Provided for non-commercial research and education use. Not for reproduction, distribution or commercial use.

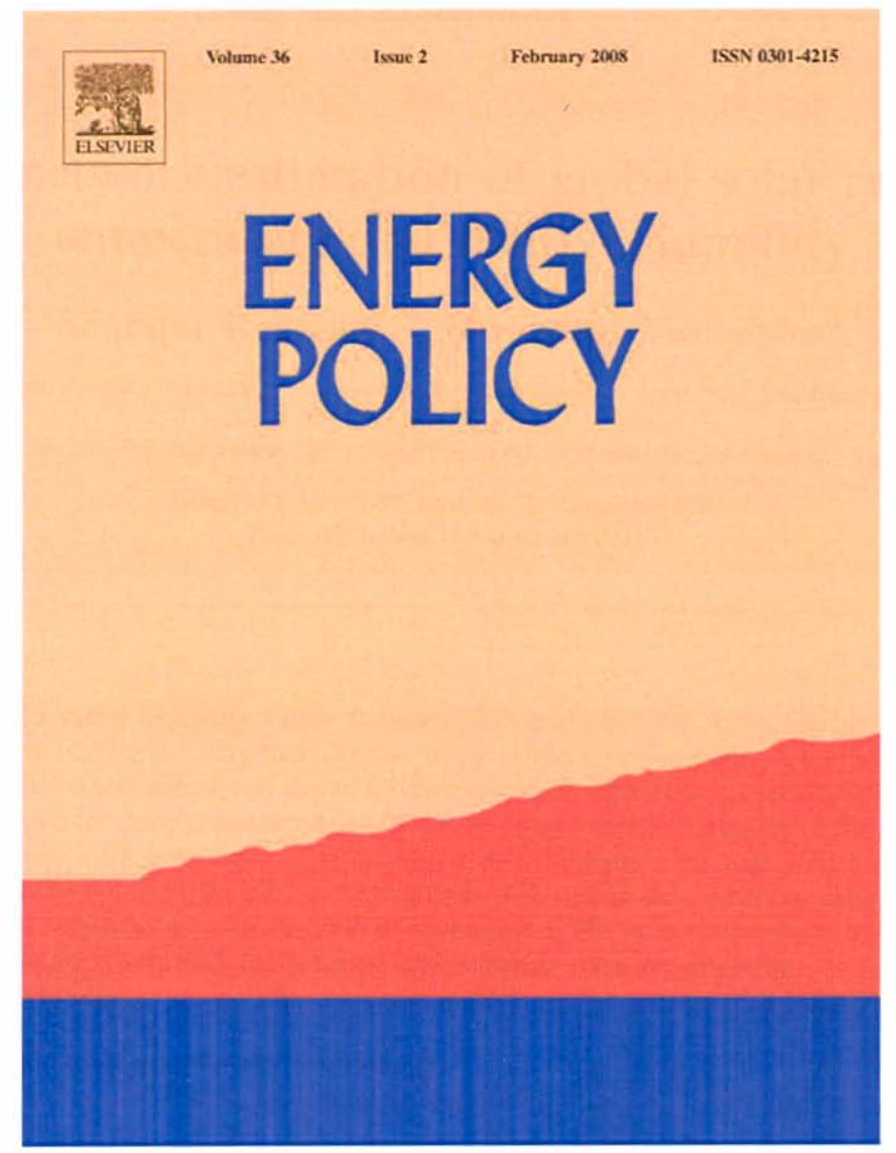

This article was published in an Elsevier journal. The attached copy

is furnished to the author for non-commercial research and education use, including for instruction at the author's institution, sharing with colleagues and providing to institution administration.

Other uses, including reproduction and distribution, or selling or licensing copies, or posting to personal, institutional or third party websites are prohibited.

In most cases authors are permitted to post their version of the article (e.g. in Word or Tex form) to their personal website or institutional repository. Authors requiring further information regarding Elsevier's archiving and manuscript policies are encouraged to visit: 


\title{
Artificial neural network estimation of global solar radiation using air temperature and relative humidity
}

\author{
Shafiqur Rehman ${ }^{\mathrm{a}, *}$, Mohamed Mohandes ${ }^{\mathrm{b}}$ \\ ${ }^{a}$ Engineering Analysis Section, Center for Engineering Research, Research Institute, King Fahd Unirersity of Petroleum and Minerals, Box \# 767, \\ Dhahran 31261, Saudi Arabia \\ ${ }^{b}$ Electrical Engineering Department, King Fahd University of Petroleum and Minerals, Saudi Arabia \\ Received 9 July 2007; accepted 26 September 2007 \\ Available online 14 November 2007
}

\begin{abstract}
Measured air temperature and relative humidity values between 1998 and 2002 for Abha city in Saudi Arabia were used for the estimation of global solar radiation (GSR) in future time domain using artificial neural network method. The estimations of GSR were made using three combinations of data sets namely; (i) day of the year and daily maximum air temperature as inputs and GSR as output, (ii) day of the year and daily mean air temperature as inputs and GSR as output and (iii) time day of the year, daily mean air temperature and relative humidity as inputs and GSR as output. The measured data between 1998 and 2001 were used for training the neural networks while the remaining 240 days' data from 2002 as testing data. The testing data were not used in training the neural networks. Obtained results show that neural networks are well capable of estimating GSR from temperature and relative humidity. This can be used for estimating GSR for locations where only temperature and humidity data are available.
\end{abstract}

(c) 2007 Elsevier Ltd. All rights reserved.

Keywords: Artificial neural networks; Meteorology; Global solar radiation

\section{Introduction}

Due to increasing costs of fossil fuels, uncertainty of availability, increasing environmental pollution and general awareness amongst the common people, the green sources of energy are being encouraged. The green sources of energy include solar photovoltaic, solar thermal wind, biomass, small and big hydro, tidal wave, ocean, etc. Among these sources of energy, wind, solar and hydro are the common ones in use. Despite being relatively costly compared to wind and other green sources of energy, solar photovoltaic technology is being used commonly for the generation electricity for both grid connected and standalone power systems. For proper, economical and efficient development and utilization of solar energy, an accurate knowledge of the availability and variability of solar radiation intensity both in time and special domain is very critical.

\footnotetext{
*Corresponding author. Tel.: + 9663 8603802; fax: + 96638603996

E-mail address: srehman@kfupm.edu.sa (S. Rehman).
}

Usually, the global solar radiation (GSR) measurements are made at few locations in a country, especially in developing countries, which mayor may not be the same as the actual site of solar energy development and utilization. In order to know the behavior of solar radiation at the site of interest, long-term data from a nearby location along with empirical, semiempirical, physical, neural networks, wavelets, fractals, etc. techniques is used. An extensive amount of work on Angstrom type of empirical models for the estimation of GSR on horizontal surface using measured sunshine duration values has been cited in the literature (for example Angstrom, 1924; Hussain, 1984; Samuel, 1991; Akinoglu and Ecevit, 1990; Rehman, 1998; Aguiar and Collares-Pereira. 1992). In recent years, neural network methods have been employed for the prediction of GSR both in time and special domains as can be seen from these references (Elizondo et a!.. 1996; AIAlawi and AI-Hinai, 1998; Mohandes et aI., 1998, 2000; Togrul and Onat, 1999; Hontoria et aI., 200 I, 2002; Tasadduq et aI., 2002; Kalogirou et aI., 2002; Tymvios et al.. 2005). 
Since the temperature and relative humidity records are more readily available around the globe, these values are being used to estimate the GSRs. Elizondo et al. (1996) used meteorological parameters such as air temperature. precipitation, clear-sky radiations, day length and day of the year as input into the feedforward neural network technique for the estimation of daily GSR. Al-Alawi and Al-Hinai (1998) predicted total radiation to an accuracy of $93 \%$ by using meteorological parameters as input into artificial neural network (ANN) models. Toĝrul and Onat (1999) used geographical and meteorological parameters along with the ANN methods for the prediction of GSR for a city in Turkey. Kalogirou et al. (2002) used a recurrent neural network method to estimate the maximum solar radiation using measured values of air temperature and relative humidity as input. The authors found that the correlation coefficient varied between $98.6 \%$ and $98.8 \%$. Sözen et al. (2004) used meteorological and geographical parameters as input into the neural networks model for the prediction solar potential for Turkey. In another study, Yang and Koike (2002) utilized upper air humidity values for the estimation of solar radiation on the surface of the earth through ANN method.

The present paper utilizes the air temperature, day of the year and relative humidity values as input in neural networks for the prediction of GSR on horizontal surfaces for Abha, a city in the western region of Saudi Arabia.

\section{Data description}

The $5 \mathrm{~min}$ mean data on air temperature, relative humidity, GSR, etc. at Abha and 11 other locations are collected since January 1998 by King Abdulaziz. City for Science and Technology. The solar radiation data collection station is situated at a latitude of $18.23 \mathrm{~N}$, longitude of $42.66 \mathrm{~W}$ and an altitude of $2039 \mathrm{~m}$ above sea level. The climate of Abha is classified as moderate with long-term temperature and relative humidity varying between 1.2 and $36{ }^{\circ} \mathrm{C}$ and $5 \%$ and $100 \%$, respectively throughout the year. The GSR was found to vary between a minimum of 0 to a maximum of $9900 \mathrm{~W} / \mathrm{m}^{2}$. Figs. $1-3$ show the variation of air temperature. relative humidity and GSR for Abha between 1998 and 2002, respectively.

The daily variation of air temperature, relative humidity and GSR is shown in Figs. 1-3, respectively. The daily average values of temperature were found to vary between approximately 10 and $27^{\circ} \mathrm{C}$ during the years 1998-2001 while in 2002 the temperature varied between 12 and $27 \mathrm{C}$, as can be seen from Fig. 1. The daily total values of GSR fluctuated approximately between 3 and $8 \mathrm{kWh} / \mathrm{m}^{2}$ day

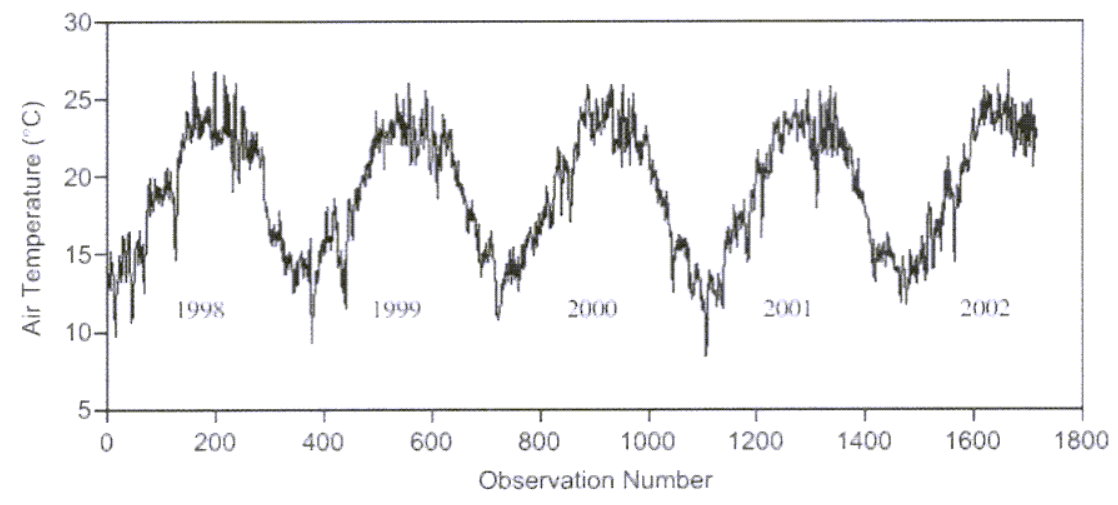

Fig. 1. Daily mean air temperature at Abha.

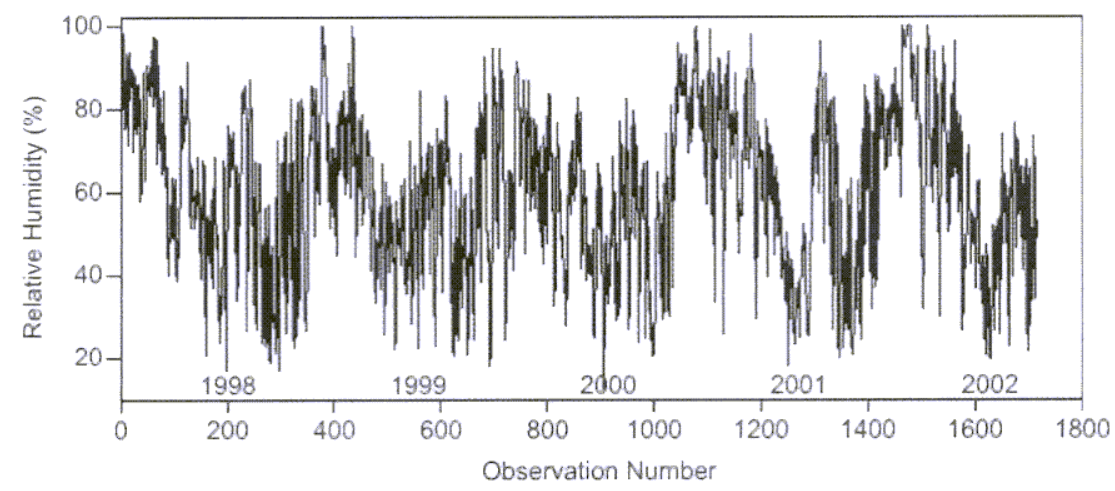

Fig. 2. Daily mean relative humidity at Abha. 


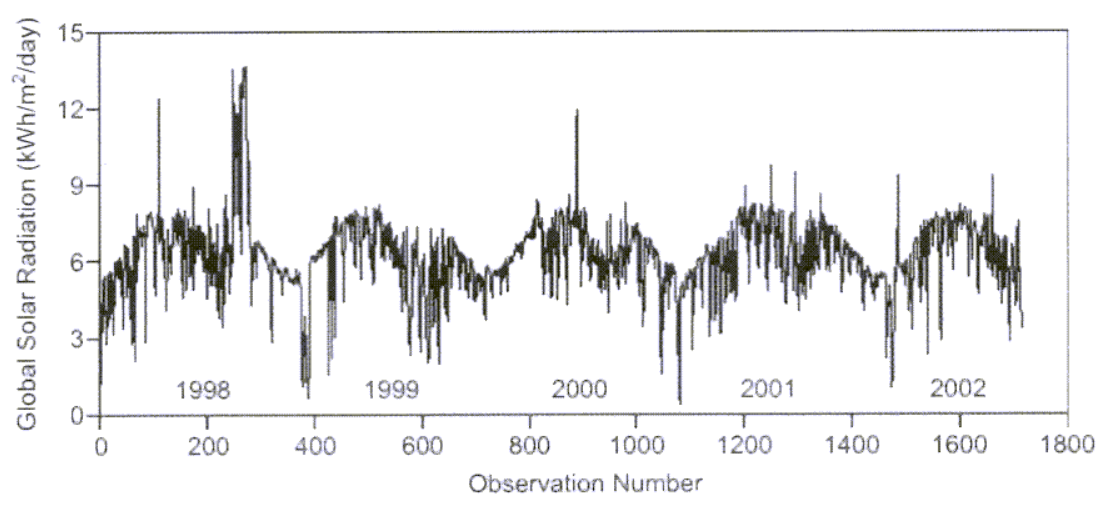

Fig. 3. Daily mean glohal solar radiation at Abhat

with some peaks reaching $12-14 \mathrm{kWh} / \mathrm{m}^{2}$ day in 1998 and 2000, as shown in Fig. 3.

\section{Methodology used}

The interest in ANNs is largely due to their ability to mimic natural intelligence in its learning from experience (Wasserman. 1993). They learn from examples by constructing an input-output mapping without explicit derivation of the model equation. ANNs have been used in a broad range of applications including: pattern classification (Lippmann, 1987; Bishop, 1996), function approximation. optimization. prediction and automatic control (Pham and Liu, 1995) and many others. Additionally, $A N N s$ have been used extensively for meteorological applications. In this paper, ANNs are used for the estimation of GSR based on measured temperature and relative humidity data.

An ANN consists of many interconnected identical simple processing units called neurons. Each connection to a neuron has an adjustable weight factor associated with it. Every neuron in the network sums its weighted inputs to produce an internal activity level $a_{i}$.

$a_{i}=\sum_{j=1}^{n} w_{i j} x_{i j}-w_{i w}$

where $w_{i j}$ is the weight of the connection from input $j$ to neuron $i, x_{i j}$ is input signal number $j$ to neuron $i$, and $w_{i 0}$ is the threshold associated with unit $i$. The threshold is treated as a normal weight with the input clamped at -1 . The internal activity is passed through a nonlinear function $\varphi$ to produce the output of the neuron $y_{i}$,

$y_{i}=\varphi\left(a_{i}\right)$.

Several forms of differentiable activation functions have been used with the most popular being the logistic function of the form:

$\varphi\left(a_{i}\right)=\frac{1}{1+\exp \left(-a_{i}\right)}$.

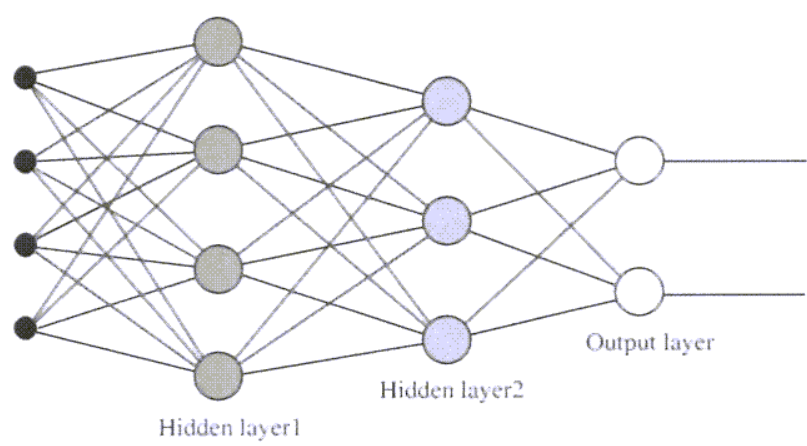

Fig. 4. Three-layer feedforward neural network.

The weights of the connections are adjusted during the training process to achieve the desired input/output relation of the network.

A multilayer feedforward network has its neurons organized into layers with no feedback or lateral connections. Layers of neurons other than the output layer are called hidden layers. The input signal propagates through the network in a forward direction, on a layer-by-layer basis. Fig. 4 shows a three-layer feedforward network.

The backpropagation algorithm (Rumelhart et al., 1986) is a supervised iterative training method for multilayer feedforward nets with sigmoidal nonlinear threshold units. It uses training data consisting of $S$ input-output pairs of vectors that characterize the problem. Using a generalized least-mean-square algorithm, the backpropagation algorithm minimizes the mean square difference between the real network output and the desired output (Haykin, 1984). The error function that the backpropagation algorithm minimizes is the average of the square difference between the output of each neuron in the output layer and the desired output. The error function can be expressed as

$E=\frac{1}{S} \sum_{s} \sum_{k}\left(d_{s k}-o_{s k}\right)^{2}$.

where $s$ is the index of the $S$ training pair of vectors, $k$ is the index of elements in the output vector, $d_{s k}$ is the $k$ th element of the sth desired pattern vector, and $o_{s k}$ is the $k$ th 
element of the output vector when pattern $s$ is presented as input to the network. Minimizing the cost function represented in Eq. (4) results in an updating rule to adjust the weights of the connections between neurons. The weight adjustment of the connection between neuron $i$ in layer $m$ and neuron $j$ in layer $m+1$ can be expressed as

$\Delta w_{j i}=\eta \delta_{j} o_{i}$

where $i$ is the index of units in layer $m, \eta$ is the learning rate, $o_{i}$ is the output of unit $i$ in the $m$ th layer, and $\delta_{j}$ is the delta error term backpropagated from the $j$ th unit in layer $m+1$ defined by

$\delta_{j}=\left[d_{j}-o_{j}\right] o_{j}\left[1-o_{j}\right]$, neuron $j$ is in the output layer,

$\delta_{j}=y_{j}\left[1-y_{j}\right] \sum_{k} \delta_{k} w_{k j}$

neuron $j$ is in a hidden layer and $k$ is index of neurons in the layer $(m+2)$, ahead of the layer of neuron $j$.

Choosing a small learning raten leads to slow rate of convergence; however, too large $\eta$ leads to oscillation. A simple method for increasing the rate of learning without oscillation is to include a momentum term as

$\Delta w_{j i}(n+1)=\eta \delta_{j} o_{i}+\alpha \Delta w_{j i}(n)$,

where $n$ is the iteration number, and $\alpha$ is a positive constant which determines the effect of past weight changes on the current direction of movement in weight space. Detailed description of the multilayer feedforward neural networks and the backpropagation algorithm may be found in Haykin (1984).

\section{Results and discussion}

First, a feedforward ANN has been trained to estimate the GSR based on the daily maximum temperature and day of the year. After several experiments it was found that a network with 2 inputs, 24 hidden neurons in one layer and one output unit was sufficient for such application. The data for 1462 days during 1998-2001 were used for training purpose, while data for 240 days from the year 2002 were used for testing the performance. A maximum of 1000 iterations was allowed. The estimated GSR values were compared with that measured for the testing period as shown in Fig. 5. The mean squared error for these data was found to be $2.823 \times 10^{-4}$, while the absolute mean percentage error was $10.3 \%$.

Another feedforward ANN was trained to estimate GSR based on the daily mean temperature and day of the year. A network of two inputs, 32 hidden neurons in one layer and one output was found to perform reasonably good for this case. With same data division as done in the previous case, the obtained mean squared error was 0.0052 while the absolute mean percentage error was $11.8 \%$. The measured and estimated values of GSR for this case are shown in Fig. 6.

Lastly, a neural network with three inputs, 24 hidden neurons in one layer and one output unit was trained on the day of the year, daily mean relative humidity and daily mean temperature to predict the GSR. The same range of training data (1462) days used for training and data for 240 days for testing. The mean squared data

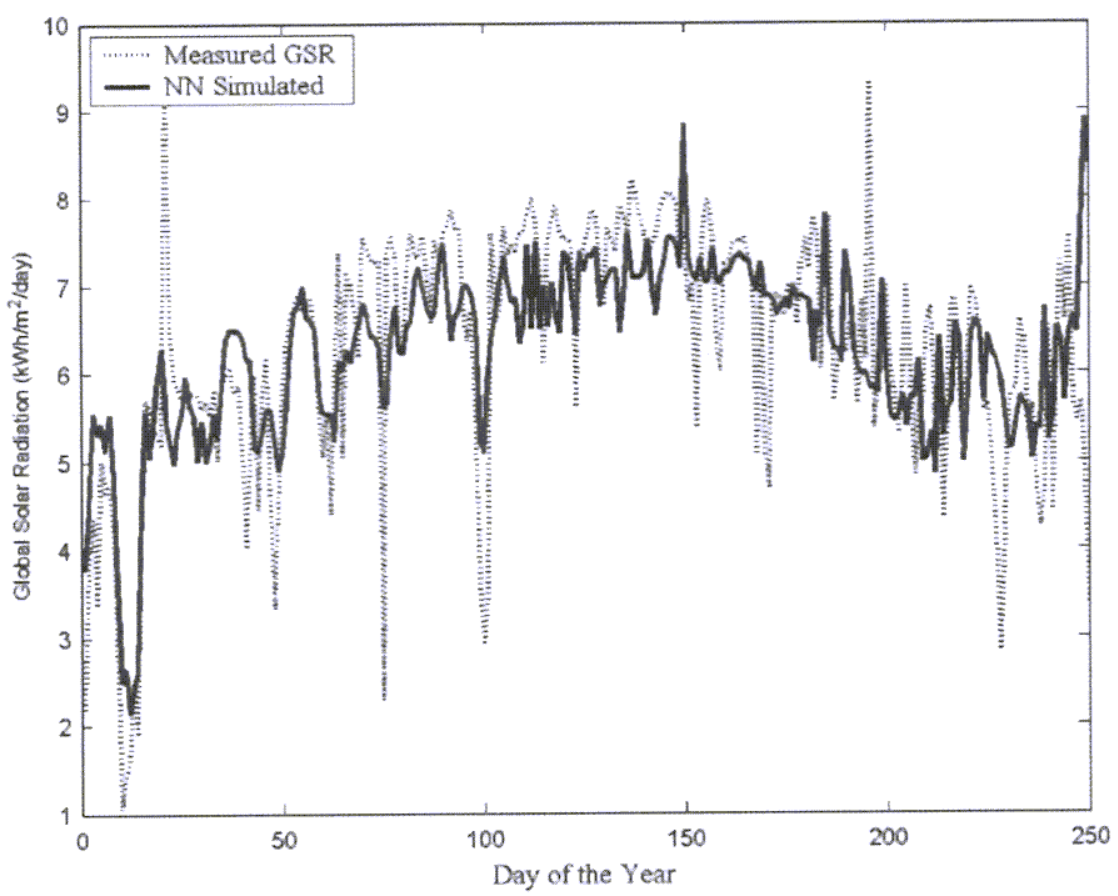

Fig. 5. Estimated and measured GSR on testing data when daily maximum temperature and day of the year are used as inputs 


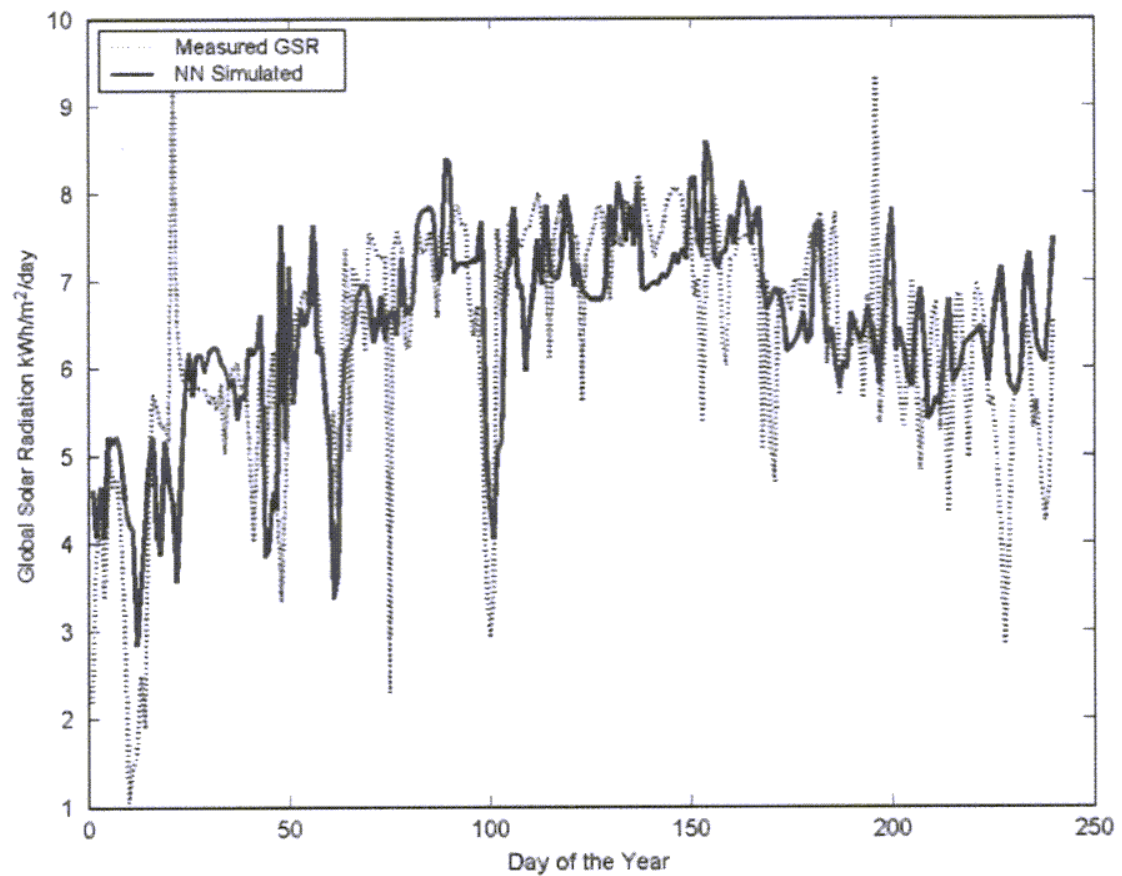

Fig. 6. Estimated and measured GSR on testing data when mean daily temperature and day of the year are used as inputs.

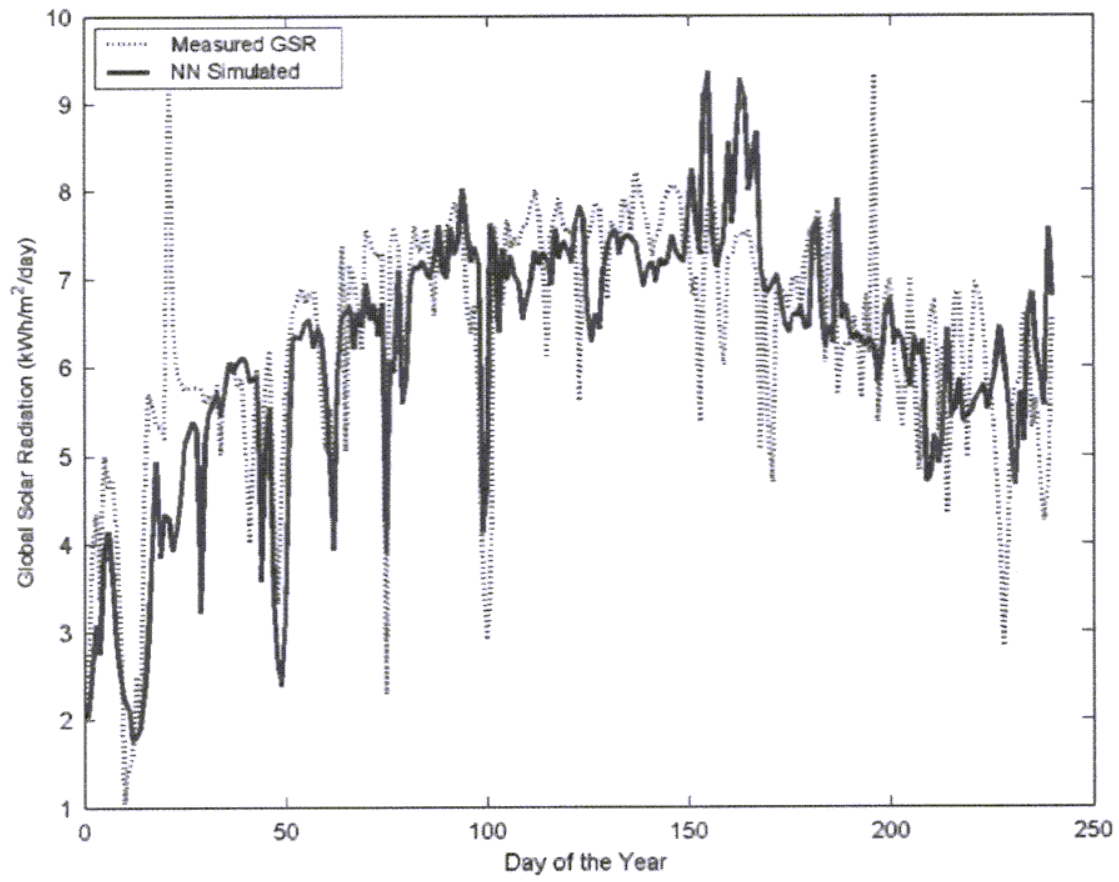

Fig. 7. Estimated and measured GSR on testing data when relative humidity, mean daily temperature and day of the year are used as inputs.

error for this case on testing data was $3.0148 \times 10^{-5}$, while the absolute mean percentage error was $4.49 \%$. Fig. 7 shows plot of the measured and estimated GSR for the testing data.

\section{Concluding remarks}

This paper presents the outcome of an attempt made to predict the GSR based on measured values of temperature 
and relative humidity only. This is important because temperature and relative humidity are commonly available parameters, while GSR is costly and requires continuous attention of skilled manpower. Data for Abha city in Saudi Arabia between 1998 and 2001 were used for training a feedforward ANN using backpropagation algorithm. Data for 240 days in the year 2002 were used to test the performance of the ANN system. For one case, only the day of the year and daily maximum temperature were used as inputs and GSR as output. In second case, the day of the year and daily mean value of temperature were used as inputs and GSR as output. In the last case, the day of the year, and daily average values of temperature and relative humidity were used to predict the GSR. Results show that using the relative humidity along with daily mean temperature outperforms the other cases with absolute mean percentage error of $4.49 \%$. The absolute mean percentage error for the case when only day of the year and mean temperature were used as inputs was $11.8 \%$ while when maximum temperature is used instead of mean temperature is $10.3 \%$. For future work. an attempt will be made to predict the diffused and normal incident solar radiation on horizontal surfaces based on meteorological parameters as well.

\section{Acknowledgment}

The authors acknowledge the support provided by the King Fahd University of Petroleum and Minerals, Dhahran, Saudi Arabia in conducting this study.

\section{References}

Aguiar, R.. Collares-Pereira, M., 1992. A time dependent autoregressive, Gaussian model for generating synthetic hourly radiation. Solar Energy 49. 167-174.

Akinoglu. B.G., Eccvit, A.. 1990. Construction of a quadratic model using modified Angstrom coefficients to cstimate global solar radiation. Solar Energy 45 (2), 85-92.

Al-Alawi. S.M., Al-Hinai, H.A., 1998. An ANN-based approach for predicting global solar radiation in locations with no measurements. Renewable Energy 14 (1-4), 199-204.

Angström. A., 1924. Solar and terrestrial radiation. Journal of the Royal Metcorological Socicty 50, 121-126.

Bishop. C.M., 1996. Neural Networks for Pattern Recognition. Oxford University Press, Oxford.
Elizondo, D.. Hoogenboom. G.. McClendon. R.. 1996. Development of a neural network to predict daily solar radiation. Agricultural and Forest Meteorology 71, 115-132.

Haykin, S.. 1984. Neural Networks: A Comprehensive Foundation. Macmillan College Publishing.

Hontoria. L., Aguilera, J.. Riesco, J., Zufiria. P.J., 2001. Recurrent neural supervised models for generating solar radiation. Journal of Intelligent \& Robotic Systems 31, 201-221.

Hontoria, L., Aguilera, J., Zufiria. P.J., 2002. Generation of hourly irradiation synthetic series using the neural network multilayer perceptron. Solar Energy 75 (2). $441-446$.

Hussain, M., 1984. Estimation of glohal and diffuse solar radiation from sunshine duration and atmospheric water vapor content. Solar Energy 33 (2). $217-220$

Kalogirou. S., Michanclides, S.. Tymbios, F., 2002. Prediction of maximum solar radiation using artificial neural networks. In: Proceedings of the WREC VII, Germany.

Lippmann, R.P.. 1987. An introduction to computing with neural nets. IEEE ASSP Magazine, 4-22.

Mohandes, M., Rehman. S. Halawani, T.O., 1998. Fstimation of global solar radiation using artificial neural networks. Renewable Energy 14 (1-4). 170-184.

Mohandes, M., Balghonaim. A., Kassas, M.. Rehman. S., Halawani. T.O. 2000. Use of radial basis functions for estimating monthly mean daily solar radiation. Solar Energy 68 (2). 161-168.

Pham, D.T., Liu, X. 1995. Neural Networks for Identification, Predication and Control. Springer, London.

Rchman, S., 1998. Solar radiation over Saudi Arabia and comparison with empirical models. Energy 23 (12), 1077-1082.

Rumelhart, D.E.. McClelland, J.L., PDP Research Group, 1986. Parallel Distributed Processing. The MIT Press. Cambridge. MA.

Samuel. T.D.M.A.. 1991. Estimation of global solar radiation for Sri Lanka. Solar Energy 47 (5), 333-337.

Sözen, A., Areaklığlu. E. ÖZalp. M., 2004. Estimation of solar potential in Turkey by artificial neural networks using meteorological and geographical data. Energy Conversion and Management 45. $3033-3052$.

Tasadduq. I., Rehman, S., Bubshait, K.. 2002. Application of neural networks for the prediction of hourly mean surface temperature in Saudi Arabia. Renewable Energy 25, 545-554.

Toğrul, I.T., Onat, E., 1999. A study for estimating the solar radiation in Elazı̄ using geographical and metcorological data. Energy Conversion and Management 40, 1577-1584.

Tymvios, F.S., Jacovides, C.P., Michaelides, S.C. Scouteli, C., 2005. Comparative study of Angström's and artificial neural networks methodologies in estimating global solar radiation. Solar Energy 78 . $752-762$.

Wasserman. P., 1993. Advanced Methods in Neural Computing. Van Nostrand Reinhold, New York.

Yang. K.. Koike, T., 2002. Estimating surface solar-radiation from upperair humidity. Solar Energy 72 (2), 177-186. 